\title{
Three Dimensional Pharmacophore Modelling of Monoamine oxidase-A (MAO-A) inhibitors
}

\author{
Kalapatapu V.V.M. Sairam ${ }^{1}$, Roop K. Khar ${ }^{1, *}$, Rama Mukherjee ${ }^{2}$ and Swatantra K. Jain ${ }^{3}$ \\ 1 Department of Pharmaceutics, Faculty of Pharmacy, Jamia Hamdard (Hamdard University), New \\ Delhi-110062, India; E-mail: kalapatapusai@gmail.com; \\ 2 Dabur Research Foundation, 22 Site IV, Sahibabad, Ghaziabad - 201010 U.P, India; \\ E-mail:ramam@dabur.com
}

3 Department of Biotechnology, Faculty of Science, Jamia Hamdard (Hamdard University), New Delhi - 110062, India; E-mail: sk608@ rediffmail.com

* Author to whom correspondence should be addressed; Tel: 91-11-26059688, 9676, 9678

Extn 5605 (O); Fax: 91-11-2605 9663; E-mail: rkkhar@jamiahamdard.ac.in, roopkhar@hotmail.com

Received: 2 May 2007; in revised form: 25 July 2007 / Accepted: 30 July 2007 /

Published: 3 September 2007

\begin{abstract}
Flavoprotein monoamine oxidase is located on the outer membrane of mitochondria. It catalyzes oxidative deamination of monoamine neurotransmitters such as serotonin, norepinephrine and dopamine and hence is a target enzyme for antidepressant drugs. MAO (mono amine oxidase) has two isoforms, namely MAO-A and MAO-B. MAO-A isoform has higher affinity for serotonin and norepinephrine, while; MAO-B preferentially deaminates phenylethylamine and benzylamine. These important properties determine the clinical importance of MAO inhibitors. Selective MAO-A inhibitors are used in the treatment of neurological disorders such as depression. In this article we have developed a Hypogen pharmacophore for a set of 64 coumarin analogs and tried to analyze the intermolecular H-bonds with receptor structure.
\end{abstract}

Keywords: MAO-A, pharmacophore, docking. 


\section{Introduction}

Monoamine oxidase is a flavoprotein located at the outer membrane of mitochondria in neuronal, glial and other cells. It catalyzes oxidative deamination of monoamine neurotransmitters such as serotonin, norepinephrine and dopamine and hence is a target enzyme for antidepressant drugs. In addition, it is also responsible for the biotransformation of 1-methyl-4-phenyl-1,2,3,6tetrahydropyridine into 1-methyl-4-phenylpyridinium a Parkinsonian producing neurotoxin [1]. MAO may also have a role in apoptotic processes.

MAO exists in two forms, namely MAO-A and MAO-B. Cloning and sequencing of MAO-A and MAO-B has great impact in understanding their molecular properties respectively. This has established the fact that both the enzymes are separate and share many similar properties such as covalent link between FAD and cysteine residue, Cys 406 in MAO-A and Cys 397 in MAO-B, through an 8 $\alpha$ (cysteinyl)-riboflavin. In spite of these similarities, two enzymes have separate but overlapping biological functions. Therefore, design of specific inhibitors would lead to little or no side effects which most of existing inhibitors suffer from.

Specific substrates and inhibitors characterize both MAO subtypes. MAO-A has a higher affinity for serotonin and norepinephrine, while; MAO-B preferentially deaminates phenylethylamine and benzylamine. These properties determine the clinical importance of MAO inhibitors. Selective MAOA inhibitors are used in the treatment of neurological disorders such as depression, whereas the MAOB inhibitors are useful in the treatment of Parkinson's and Alzheimer's disease. In the light of these facts, we ventured into developing a pharmacophore for a set of 64 coumarin analogs [2,3], with close comparisons from structure based interactions.

\section{Computational Method}

The three-dimensional pharmacophore was developed using the CATALYST 4.1 software [4]. This is an integrated commercially available software package that generates pharmacophores, commonly referred to as hypotheses. It enables the use of structure and activity data for a set of lead compounds to create a hypothesis, thus characterizing the activity of the lead set. At the heart of the software is the HypoGen algorithm that allows identification of hypotheses that are common to the "active" molecules in the training set but at the same time not present in the "inactives". Structures of 64 monoamine oxidase inhibitors [2,3] of the training and test set were built and energy minimized using smart minimize [5] module from the Cerius $^{2}$. The CATALYST model treats molecular structures as templates comprising chemical functions localized in space that will bind effectively with complementary functions on the respective binding proteins. The most relevant chemical features are extracted from a small set of compounds that cover a broad range of activity. The best searching procedure was applied to select representative conformers within $20 \mathrm{kcal} / \mathrm{mol}$ from the global minimum. The conformational model of the training set was used for hypothesis (pharmacophore) generation within CATALYST, which aims to identify the best 3-dimensional arrangement of chemical functions explaining the activity variations among the compounds in the training set. The automatic generation procedure using the HypoGen algorithm in CATALYST was adopted for generation of the hypotheses. In order to obtain a reliable model, which adequately describes the interaction of ligands 
with high predictability, the method recommends a collection of training set with biological activity covering 4-5 orders of magnitude for the training set.

The pharmacophore/hypotheses are described by a set of functional features such as hydrophobic, hydrogen-bond donor, hydrogen-bond acceptor, and positive and negative ionizable sites distributed over a 3D space. The hydrogen-bonding features are vectors, whereas all other functions are points. The statistical relevance of the obtained hypotheses is assessed on the basis of their cost relative to the null hypothesis and their correlation coefficient.

Pharmacophore generation was carried out with the 64 monoamine oxidase-A inhibitors (Table 1) by setting the default parameters in the automatic generation procedure in CATALYST such as function weight 0.302 , mapping coefficient 0 , resolution $10 \mathrm{pm}$ (due to smaller size of the inhibitor set considered for the study), and activity uncertainty 3 . As uncertainty $\Delta$ in the CATALYST paradigm indicates an activity value lying somewhere in the interval from "activity divided by $\Delta$ " to "activity multiplied by $\Delta$ ". Hypotheses approximating the pharmacophore of the MAO-A inhibitors are described as a set of aromatic hydrophobic, hydrogen-bond acceptor, hydrogen bond acceptor lipid, positively and negatively ionizable sites distributed within a 3D space. The statistical relevance of various generated hypothesis is assessed on the basis of the cost relative to the null hypothesis and the correlation coefficients. The hypothesis is then used to estimate the activities of the training set. These activities are derived from the best conformation generation mode of the conformers displaying the smallest root-mean-square (RMS) deviations when projected onto the hypothesis. HypoGen considers a pharmacophore that contain features with equal weights and tolerances. Each feature (e.g., hydrogenbond acceptor, hydrogen-bond donor, hydrophobic, positive ionizable group, etc.) contributes equally to estimate the activity. Similarly, each chemical feature in the HypoGen pharmacophore requires a match to a corresponding ligand atom to be within the same distance (tolerance). Thus, two parameters such as the fit score and conformational energy costs are crucial for estimation of predicted activity of the compounds.

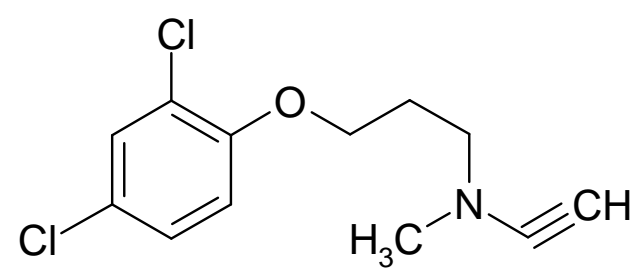

\section{Scheme 1.}

Docking. The crystal structure of monoamine oxidase A with clorgyline (Scheme 1) was used in the study. Protein was prepared as per standard protocol. Docking was carried out using GOLD, which used the genetic algorithm (GA) with default values. Docking was carried out using GOLD, which uses the genetic algorithm (GA). For each of the 10 independent GA runs, a maximum number of 100,000 GA operations were performed on a set of five groups with a population size of 100 individuals. Operator weights for crossover, mutation, and migration were set to 95, 95, and 10, respectively. Default cutoff values of $2.5 \AA(d \mathrm{H}-\mathrm{X})$ for hydrogen bonds and $4.0 \AA$ for van der Waals were employed. Hydrophobic fitting points were calculated to facilitate the correct starting orientation of the ligand for 
docking by placing the hydrophobic ligand atoms appropriately in the corresponding areas of the active site. When the top three solutions attained root-mean-square deviation (RMSD) values within $1.5 \AA$, GA docking was terminated. The first ranked solutions of the ligands were taken for further analysis.

\section{Results and Discussion}

We developed a HypoGen 3D-QSAR pharmacophore model for monoamine oxidase-A inhibitory activity from a set of 64 inhibitors [2,3] (Table I). These compounds display a broad range of inhibitory activity against MAO-A with experimental $\mathrm{IC}_{50}$ values ranging from $2.0 \times 10^{-8}$ to $1.0 \times 10^{-4}$ (Table II). On the basis of the structure-activity relationships within this set of compounds, pharmacophore models of MAO-A inhibition were generated using the CATALYST procedure. The best model produced by CATALYST consisted of the spatial arrangement of five functional groups: two hydrogen-bond acceptors, three hydrophobic groups (Figure 1). The model shows a good correlation $(R$ $=0.95$ ) between experimental and predicted inhibitory activity of the MAO-A inhibitors of the training set. The CATALYST procedure resulted in the generation of 10 alternative pharmacophores describing the MAO-A inhibitory activity of the training set compounds. These pharmacophore models were then evaluated by using them to estimate the inhibitory activity of the training set compounds. The correlation between the estimated and experimental values ranges between 0.95 and 0.66 , and the RMS value is 1.2. The statistical significance of the pharmacophores (hypotheses) falls within the recommended range of values in CATALYST. The difference between the fixed and the null cost is found to be 54.4 bits, indicating the robustness of the correlation. The cost difference between the first and the tenth hypothesis is 13.294 bits, closer to the fixed costs than the null costs. All these calculated cost differences were found to be well within the recommended acceptable limits in the cost analysis of the CATALYST procedure. The best pharmacophore model is characterized by two hydrogen bond acceptor functions, three hydrophobic functions (Figure 1) and is also statistically the most relevant model. 
Table 1. All the molecules in the training and test set are shown. Test set molecules are given in bold face.<smiles></smiles>

$\mathrm{SS} 1-13, \mathrm{SS} 16-22$

(n)


Int. J. Mol. Sci. 2007, 8

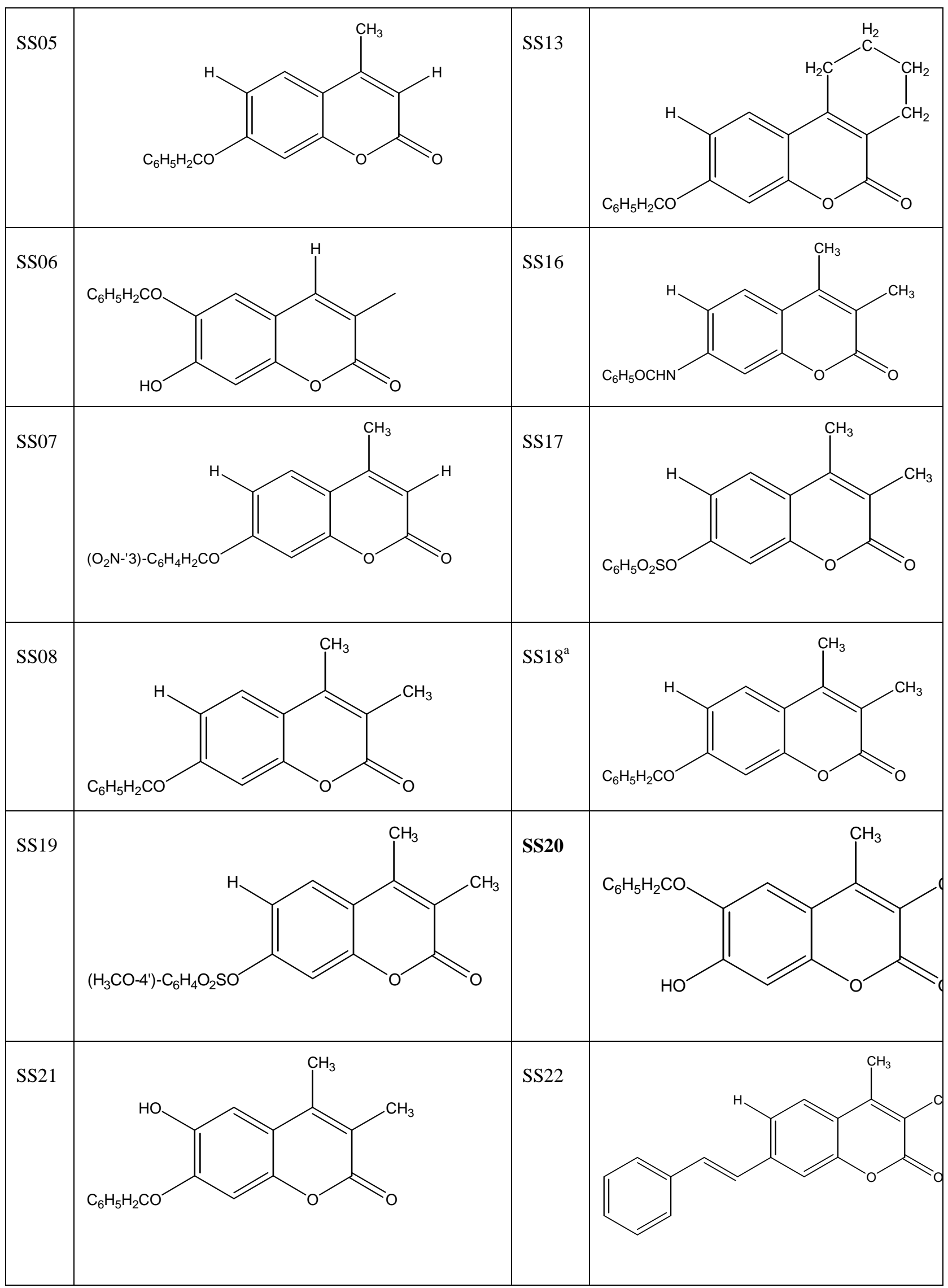

a 8-Methyl derivative 
Int. J. Mol. Sci. 2007, 8

Table 1 continued.
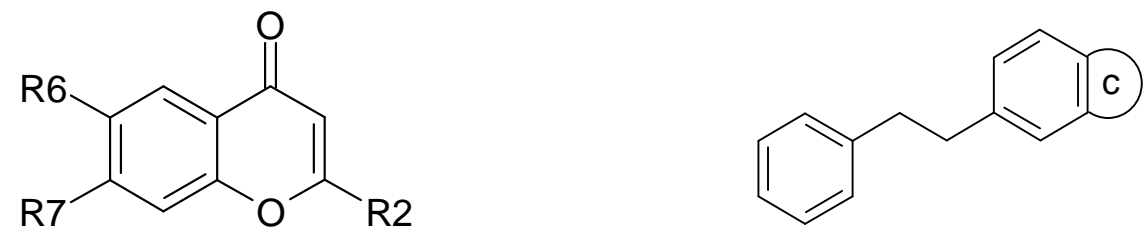

SS $14,15,23$

SS24 - 26

No. Structures


Int. J. Mol. Sci. 2007, 8

Table 1 continued.

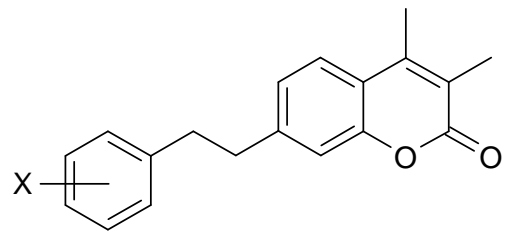

SS27 - 49

(n)


Int. J. Mol. Sci. 2007, 8

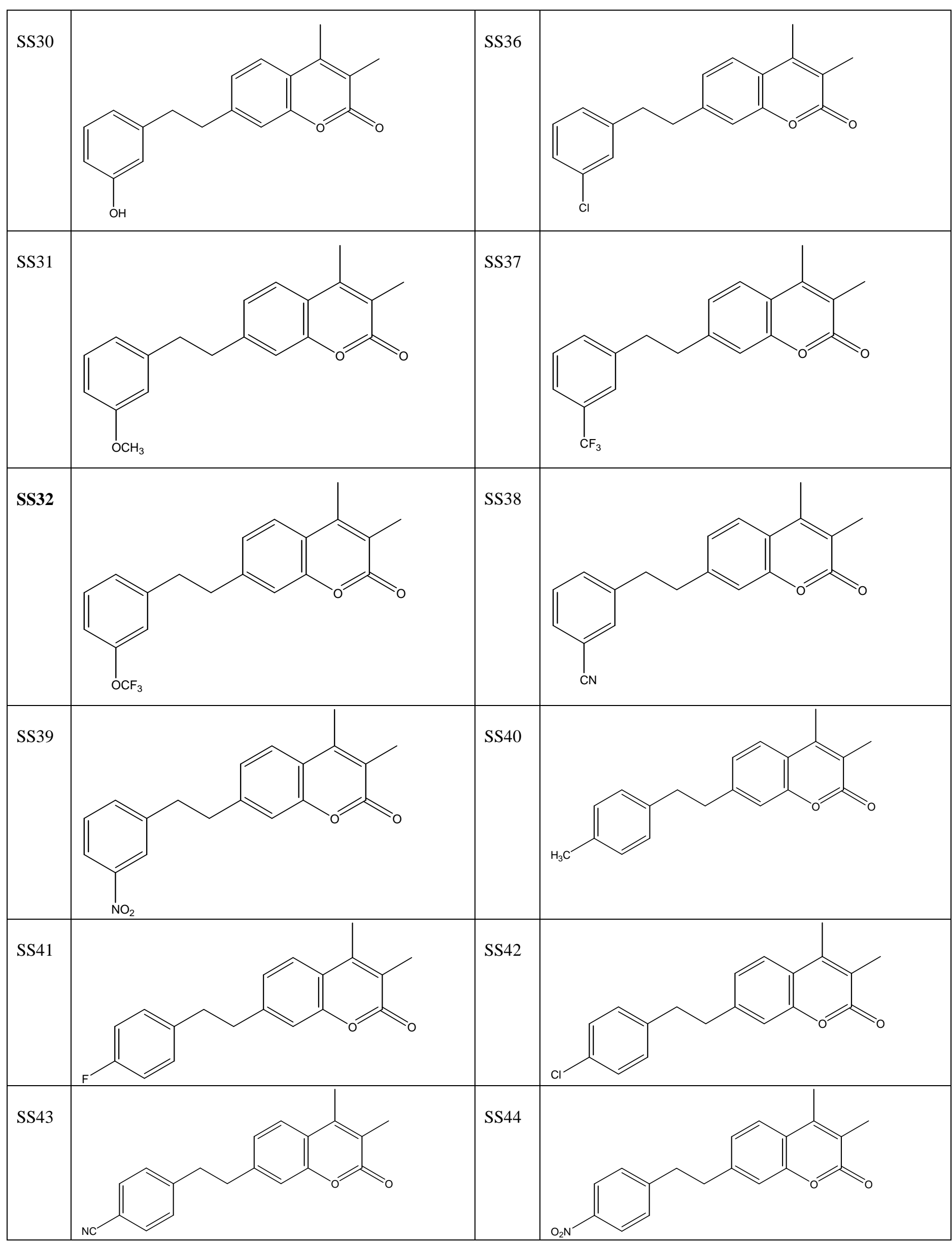


Int. J. Mol. Sci. 2007, 8

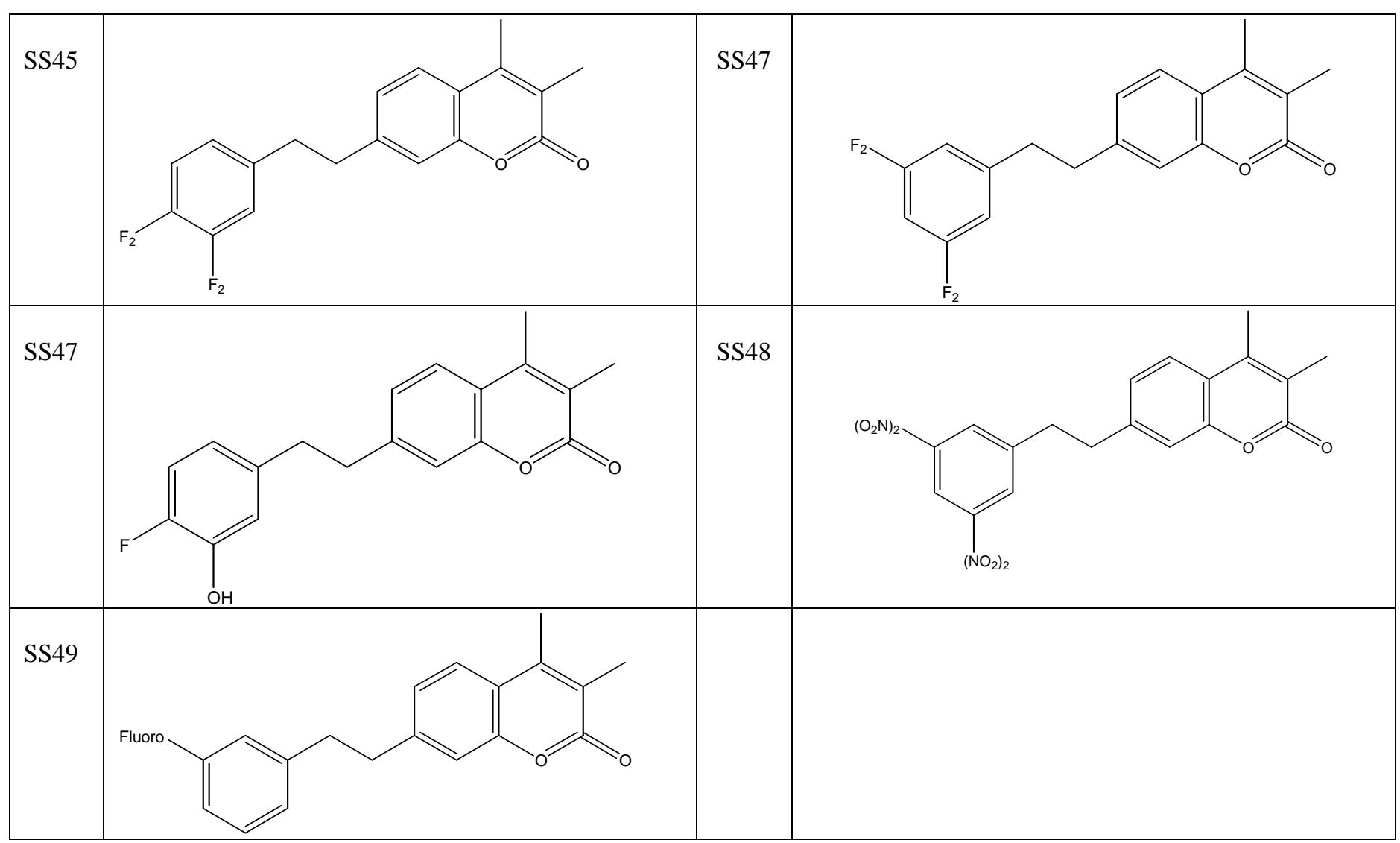

Table 1 continued.<smiles>[R]c1ccc2oc(=O)c(C([R])[R])cc2c1</smiles>

SS50 - 64

Structures



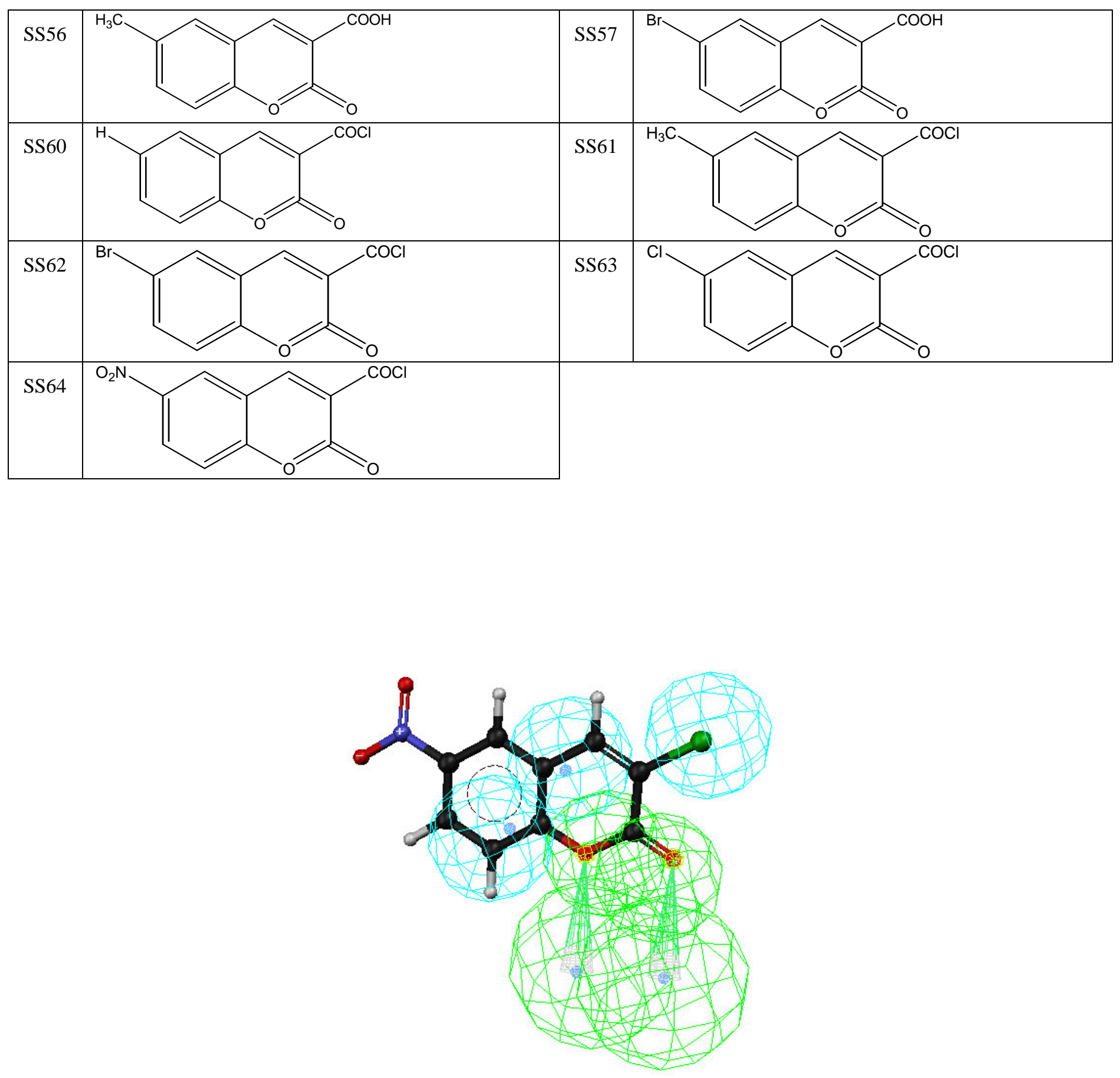

Figure 1: Active molecule 64 fitted to the pharmacophore model developed.

The estimated activity values along with the experimental $\mathrm{IC}_{50}$ values for MAO-A inhibition are presented in Table II. Experimentally determined $\mathrm{IC}_{50}$ values versus the calculated activities demonstrate a good correlation $(R=0.95)$ within the range of uncertainty 3 , indicating a good predictive power of the model. The most potent compound in the training set, molecule 62 maps well to the functional features of the pharmacophore with all five features of the molecule mapped well with hypotheses generated, whereas the least potent members of the series, molecule 26, maps poorly with the pharmacophore with only four of five features are mapped missing out the second acceptor feature 
(Figure $2 \mathrm{a}, \mathrm{b}$ ). Inspection of Figure $2 \mathrm{~B}$ clearly shows that molecule 26 fails to map the second hydrogen bond acceptor feature of the pharmacophore. Thus, it appears that the second hydrogen bond acceptor feature may be specific requirement for binding to MAO-A.

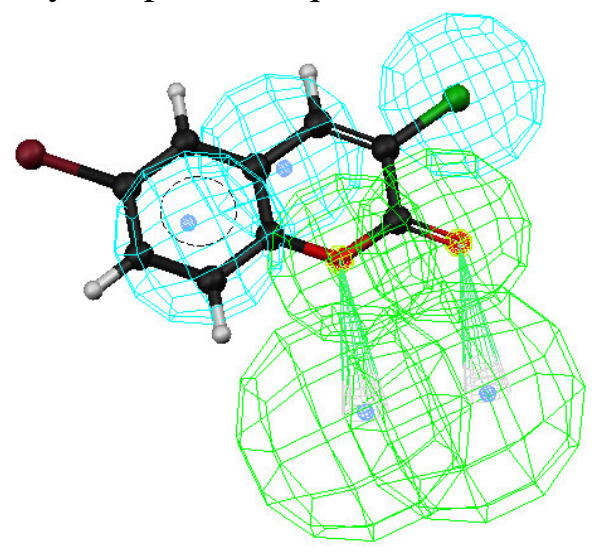

2A

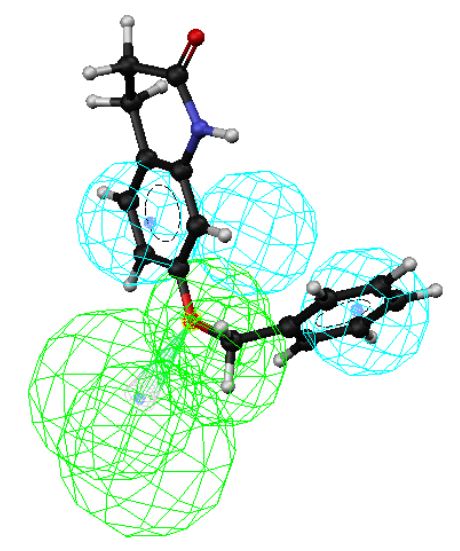

2B

Figure 2: A) Active molecule 64 in training set B) Low active molecule 26 in the training test set.

The test set considered contains three molecules 20,32 and 34. Activity predicted for the test is also found deviated. Analysis of these test set molecules suggests that bulky substitutions in the 6 and 7 positions of the coumarin ring could be responsible for this deviation in their activity. Estimated activities were calculated by scoring the pharmacophore model on the test set and comparing with the experimental $\mathrm{IC}_{50}$ values.

\section{Enrichment factor analysis}

The enrichment factor "ideal" for a set of 125 dataset of compounds that contain 24 monoamine oxidase specific inhibitors and 101 nonspecific inhibitors is 5.35. Of these 24 specific monoamine oxidase inhibitors the pharmacophore generated has picked 17 molecules and within 100 nonspecific inhibitors dataset, model has picked six molecules. Hence enrichment factor calculated over this model generated to 125 dataset is around 4. These numbers speak of evaluation of the model and ability to pick the MAO specific inhibitors in the dataset. Details of 125 dataset are provided in the supporting information.

Docking. Coumarin derivatives were docked into the active site of MAO-A and docking analysis was performed (Figure 3). It was observed that a $\pi$ interaction with Phe 407 seems to be crucial in its bound ligand clorgyline as well as coumarin analogs docked ${ }^{7}$. Clorgyline methyl group has a $\mathrm{CH} \cdots \pi$ interaction with phenyl ring of Tyr 407 and $\mathrm{CH} \cdots \mathrm{N}$ interaction with $\mathrm{N} 1$ of FAD (cofactor). This aromatic sandwich with Tyr 407 is important in stabilizing substrate within active site and is also crucial for MAO-A catalytic activity [1]. Pharmacophore model generated has three hydrophobic points which also confirms that aromatic interactions to be very important for stabilizing interactions. Santana et al., in their recent report on the QSAR of coumarin analogs confirms that hydrophobicity 
along with polarizability to be important physical factor [6]. One of the highest active molecule 64 has a strong $\mathrm{OH} \cdots \mathrm{O}$ interaction between $-\mathrm{OH}$ group of Tyr 197 and oxygen of $-\mathrm{NO}_{2}$, two weak $\mathrm{CH} \cdots \mathrm{O}$ and one $\mathrm{CH} \cdots \mathrm{N}$ interactions with Tyr 197 and FAD (phenyl $\mathrm{CH}$ of ligand has a $\mathrm{CH} \cdots \mathrm{O}$ interaction with hydroxy oxygen of Tyr 197, $\mathrm{CH} \cdots \mathrm{N}$ with $\mathrm{N} 1$ of FAD and another $\mathrm{CH} \cdots \mathrm{O}$ between oxygen of nitro group of the ligand with $\mathrm{CH}$ of $\mathrm{FAD}$ ). Lowest active molecule 4 still maintains a $\pi \cdots \pi$ interaction with Tyr 407. This molecule has very fewer interactions and perhaps the low activity can be attributed to this lack of H-bond interactions.

Table II: All the molecules in the training and test set with observed and estimated activities are shown. All test set molecules are given in bold.

\begin{tabular}{|c|c|c|c|c|c|}
\hline Sl. No. & Observed Activity & Estimated Activity & Sl. No. & Observed Activity & Estimated Activity \\
\hline 1 & 0.0000407 & 0.000039 & 33 & 0.00000588 & 0.00000037 \\
\hline 2 & 0.00000676 & 0.000021 & 34 & 0.000001 & 0.000000064 \\
\hline 3 & 0.000000389 & 0.000022 & 35 & 0.00000707 & 0.00000045 \\
\hline 4 & 0.0000417 & 0.00002 & 36 & 0.000000575 & 0.00000044 \\
\hline 5 & 0.00000195 & 0.0000022 & 37 & 0.00000122 & 0.00000029 \\
\hline 6 & 0.00000195 & 0.000018 & 38 & 0.00000191 & 0.00000011 \\
\hline 7 & 0.0000234 & 0.00000031 & 39 & 0.000000218 & 0.00000026 \\
\hline 8 & 0.000000126 & 0.0000022 & 40 & 0.0000000758 & 0.00000012 \\
\hline 9 & 0.000000192 & 0.0000023 & 41 & 0.00000371 & 0.00000021 \\
\hline 10 & 0.00000158 & 0.00000041 & 42 & 0.000000123 & 0.000000093 \\
\hline 11 & 0.000001 & 0.000002 & 43 & 0.000000123 & 0.00000011 \\
\hline 12 & 0.00000355 & 0.0000014 & 44 & 0.0000001 & 0.00000036 \\
\hline 13 & 0.00000158 & 0.0000016 & 45 & 0.000000831 & 0.00000044 \\
\hline 14 & 0.00000158 & 0.00003 & 46 & 0.000000123 & 0.00000065 \\
\hline 15 & 0.0000162 & 0.000026 & 47 & 0.000000676 & 0.0000002 \\
\hline 16 & 0.00000676 & 0.0000014 & 48 & 0.000000114 & 0.00000059 \\
\hline 17 & 0.00000138 & 0.00000036 & 49 & 0.000000181 & 0.00000045 \\
\hline 18 & 0.0000000758 & 0.0000026 & 50 & 0.000000691 & 0.00000031 \\
\hline 19 & 0.000000562 & 0.00000018 & 51 & 0.000000229 & 0.00000027 \\
\hline 20 & 0.0000000707 & 0.000000096 & 52 & 0.00000019 & 0.0000002 \\
\hline 21 & 0.000112 & 0.0000022 & 53 & 0.000000208 & 0.0000002 \\
\hline 22 & 0.00000933 & 0.0000023 & 54 & 0.000000501 & 0.00000018 \\
\hline 23 & 0.000000407 & 0.000012 & 55 & 0.000000257 & 0.000039 \\
\hline 24 & 0.0000218 & 0.000027 & 56 & 0.0000151 & 0.00003 \\
\hline 25 & 0.0000398 & 0.000023 & 57 & 0.000012 & 0.00003 \\
\hline 26 & 0.00000363 & 0.000027 & 60 & 0.0000000676 & 0.00000007 \\
\hline 27 & 0.000102 & 0.00000013 & 61 & 0.0000000602 & 0.000000039 \\
\hline 28 & 0.00000229 & 0.00000046 & 62 & 0.0000000288 & 0.000000037 \\
\hline 29 & 0.000000416 & 0.00000025 & 63 & 0.0000000199 & 0.000000036 \\
\hline 30 & 0.00000331 & 0.00000034 & 64 & 0.0000000301 & 0.000000016 \\
\hline 31 & 0.000000416 & 0.00000024 & & & \\
\hline 32 & 0.00000151 & 0.000000066 & & & \\
\hline
\end{tabular}



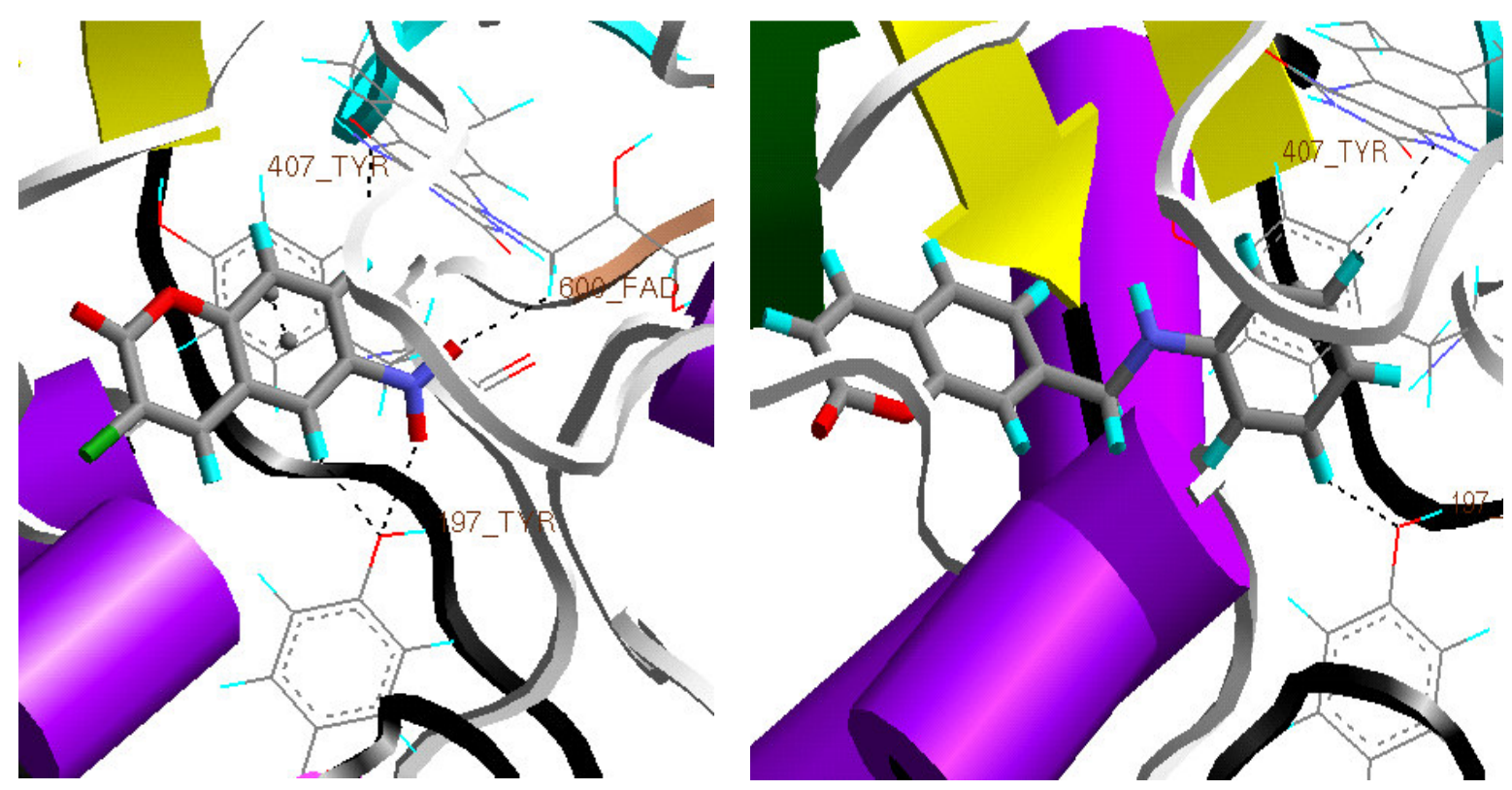

Figure 3: Left figure shows the one of the highest active molecule 64 and interactions with active site amino acids, while figure on the right side shows one of the lowest active molecule 4 in the monoamine oxidase-A active site and fewer weak hydrogen bond interactions with Tyr197 and FAD cofactor.

\section{Conclusion}

This study suggests that it would be difficult for the active site with $7 \AA$ width [7] to accommodate bigger molecules, in this case molecules 32 and 34. The same is being reflected in the phamacophore hypothesis. This study partly tries to address the inactivity of the some of the coumarin analogs using analog based methods like pharmacophore hypothesis and structure based methods like docking. A detailed study on each class of compounds would throw light on the optimization aspect in a series of analogs and structural requirements in order to have optimum binding. Understanding of topology and H-bond interactions aiding molecular recognition with receptor govern the biological activity of this set of molecules.

\section{Acknowledgement}

KVVMS thanks CSIR for financial support and Prof. G. R. Desiraju, School of Chemistry, University of Hyderabad, Hyderabad, India for encouragement and guidance.

\section{Reference}

1. Geha, R.M.; Chen, K.; Wouters, J.; Ooms, F.; Shih, J.C. Analysis of conserved active site residues in monoamine oxidase $\mathrm{A}$ and $\mathrm{B}$ and their three-dimensional molecular modeling. $J$. Biol. Chem. 2002, 277, 17209-17216. 
2. Gnerre, C.; Catto, M.; Leonetti, F.; Weber, P.; Carrupt, P.A.; Altomare, C.; Carotti, A.; Testa, B. Inhibition of monoamine oxidases by functionalized coumarin derivatives: biological activities, QSARs, and 3D-QSARs. J. Med. Chem. 2000, 43, 4747-4758.

3. Chimenti, F.; Secci, D.; Bolasco, A.; Chimenti, P.; Granese, A .M; Befani, O.; Turini, P.; Alcaro, S.; Ortuso, F. Inhibition of monoamine oxidases by coumarin-3-acyl derivatives: biological activity and computational study. Bioorg. Med. Chem. Lett. 2004, 14, 3697-3703.

4. CATALYST, Accelrys Inc. San Diego, CA.

5. Cerius ${ }^{2}$, Accelrys Inc. San Diego, CA.

6. Santana, L.; Uriarte, E.; Diaz, H. G.; Zagotto, G.; Otero, R.S.; Alvarez, E.M. A QSAR model for in silico screening of MAO-A inhibitors. Prediction, synthesis, and biological assay of novel coumarins. J. Med. Chem. 2006, 49, 1149-1156.

7. Parada, M.R.; Fierro, A.; Vasquez, P. I.; Cassels, B.K. A QSAR model for in silico screening of MAO-A inhibitors. Prediction, synthesis, and biological assay of novel coumarins. Curr. Enz. Inhib. 2005, 1, 85-95.

(C) 2007 by MDPI (http://www.mdpi.org). Reproduction is permitted for noncommercial purposes.

\section{Supplementary Information}

Table S1: Following table contains the list of compounds and citation used as a database to find out enrichment factor (measure of validation of the model).

\begin{tabular}{|l|l|l|l|}
\hline 1 & \multicolumn{1}{|c|}{ Reference } \\
\hline 2 & Structure & $\begin{array}{l}\text { Eur. J. Med. Chem. 1995, } \\
30,471-482\end{array}$ \\
\hline 3 & &
\end{tabular}




\begin{tabular}{|c|c|c|c|c|}
\hline 5 & & 5 & $\begin{array}{l}\text { J. Med. Chem } \\
\text { 3705-3713 }\end{array}$ & 1992, 35, \\
\hline 6 & & 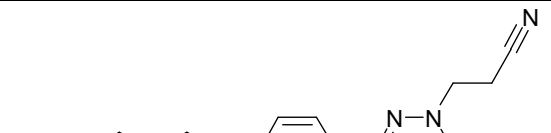 & $\begin{array}{l}\text { J. Med. Chem } \\
1157-1167\end{array}$ & 1993, 36, \\
\hline 7 & & 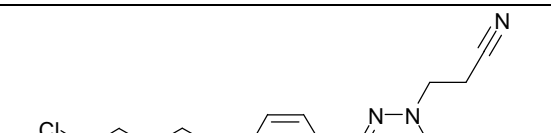 & $\begin{array}{l}\text { J. Med. Chem } \\
1157-1167\end{array}$ & 1993, 36, \\
\hline 8 & & & $\begin{array}{l}\text { J. Med. Chem } \\
1157-1167\end{array}$ & 1993, 36, \\
\hline 9 & & & $\begin{array}{l}\text { J. Med. Chem } \\
1157-1167\end{array}$ & 1993, 36, \\
\hline 10 & & & $\begin{array}{l}\text { J. Med. Chem } \\
4786-4792\end{array}$ & 1995, 38, \\
\hline 11 & $\begin{array}{l}\text { MAOA } \\
\text { Specifi } \\
\text { c }\end{array}$ & & $\begin{array}{l}\text { J. Med. Chem } \\
3455-3461\end{array}$ & 2004, 47, \\
\hline 12 & & & $\begin{array}{l}\text { J. Med. Chem } \\
\text { 2085-2089 }\end{array}$ & 1994, 37, \\
\hline 13 & & & $\begin{array}{l}\text { J. Med. Chem } \\
\text { 2085-2089 }\end{array}$ & 1994, 37, \\
\hline 14 & & & $\begin{array}{l}\text { J. Med. Chem } \\
\text { 2085-2089 }\end{array}$ & 1994, 37, \\
\hline
\end{tabular}




\begin{tabular}{|c|c|c|c|}
\hline 15 & & & $\begin{array}{l}\text { J. Med. Chem. 1993, 36, } \\
1157-1167\end{array}$ \\
\hline 16 & & & $\begin{array}{l}\text { J. Med. Chem. 1991, 34, } \\
\text { 2931-2933 }\end{array}$ \\
\hline 17 & & & $\begin{array}{l}\text { J. Med. Chem. 1991, 34, } \\
\text { 2931-2933 }\end{array}$ \\
\hline 18 & & & $\begin{array}{l}\text { J. Med. Chem. 2005, 48, } \\
2407-2419\end{array}$ \\
\hline 19 & & & $\begin{array}{l}\text { Bioorg. Med. Chem. Lett. } \\
\text { 1994, 4, 1195-1198 }\end{array}$ \\
\hline 20 & & & $\begin{array}{l}\text { J. Med. Chem. 1998, 41, } \\
2118-2125\end{array}$ \\
\hline 21 & & & $\begin{array}{l}\text { J. Med. Chem. 1998, 41, } \\
2118-2125\end{array}$ \\
\hline 22 & & & $\begin{array}{l}\text { Eur. J. Med. Chem. 1999, } \\
34,137-151\end{array}$ \\
\hline 23 & & & $\begin{array}{l}\text { J. Med. Chem. 1997, 40, } \\
2466-2473\end{array}$ \\
\hline 24 & & & $\begin{array}{l}\text { J. Med. Chem.1996, 39, } \\
\text { 1857-1863 }\end{array}$ \\
\hline 25 & Mixed & & $\begin{array}{l}\text { J. Med. Chem.1996, 39, } \\
\text { 1857-1863 }\end{array}$ \\
\hline 26 & & & $\begin{array}{l}\text { J. Med. Chem. 2002, 45, } \\
5260-5279\end{array}$ \\
\hline 27 & & & $\begin{array}{l}\text { J. Med. Chem. 1994, 37, } \\
\text { 2085-2089 }\end{array}$ \\
\hline
\end{tabular}




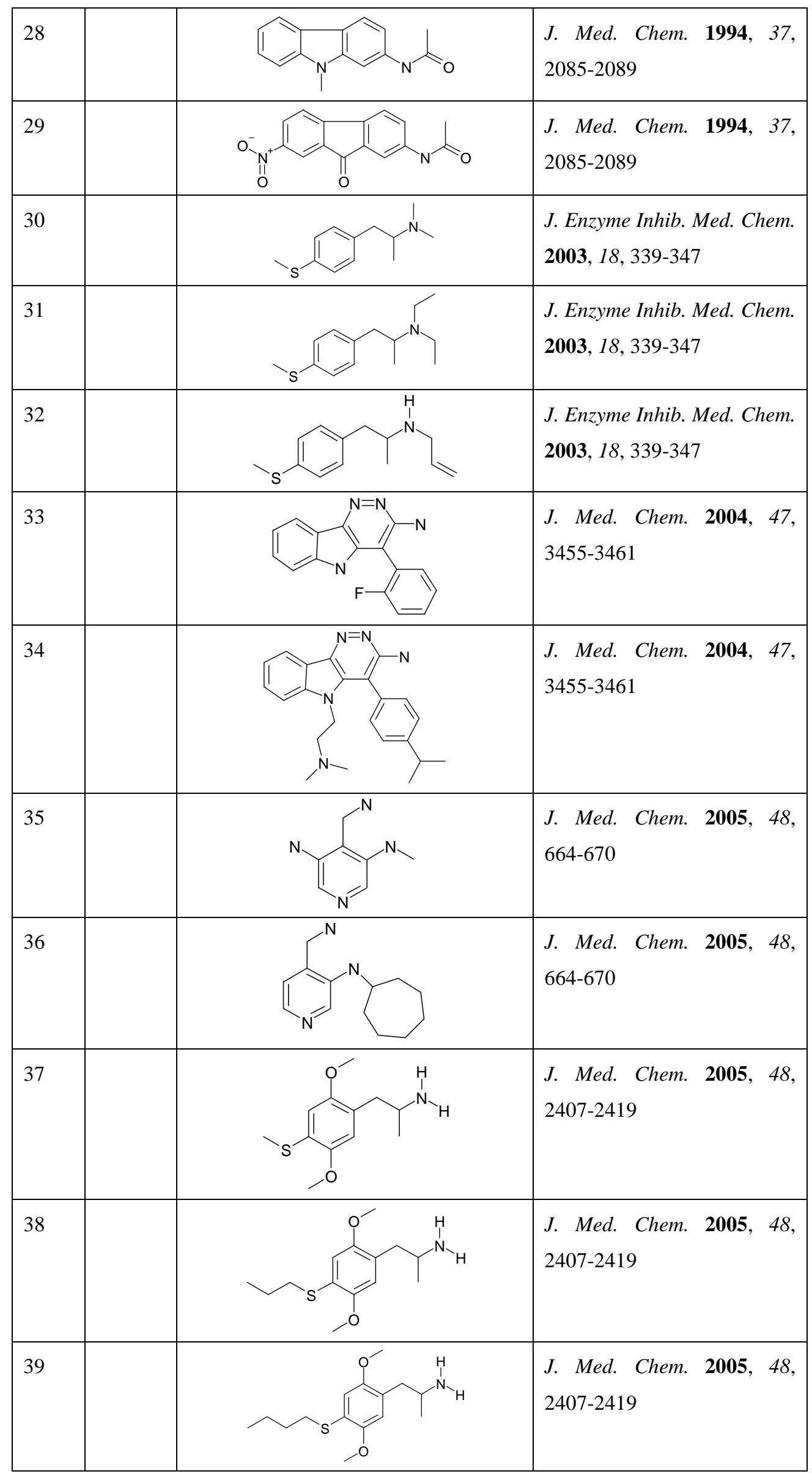




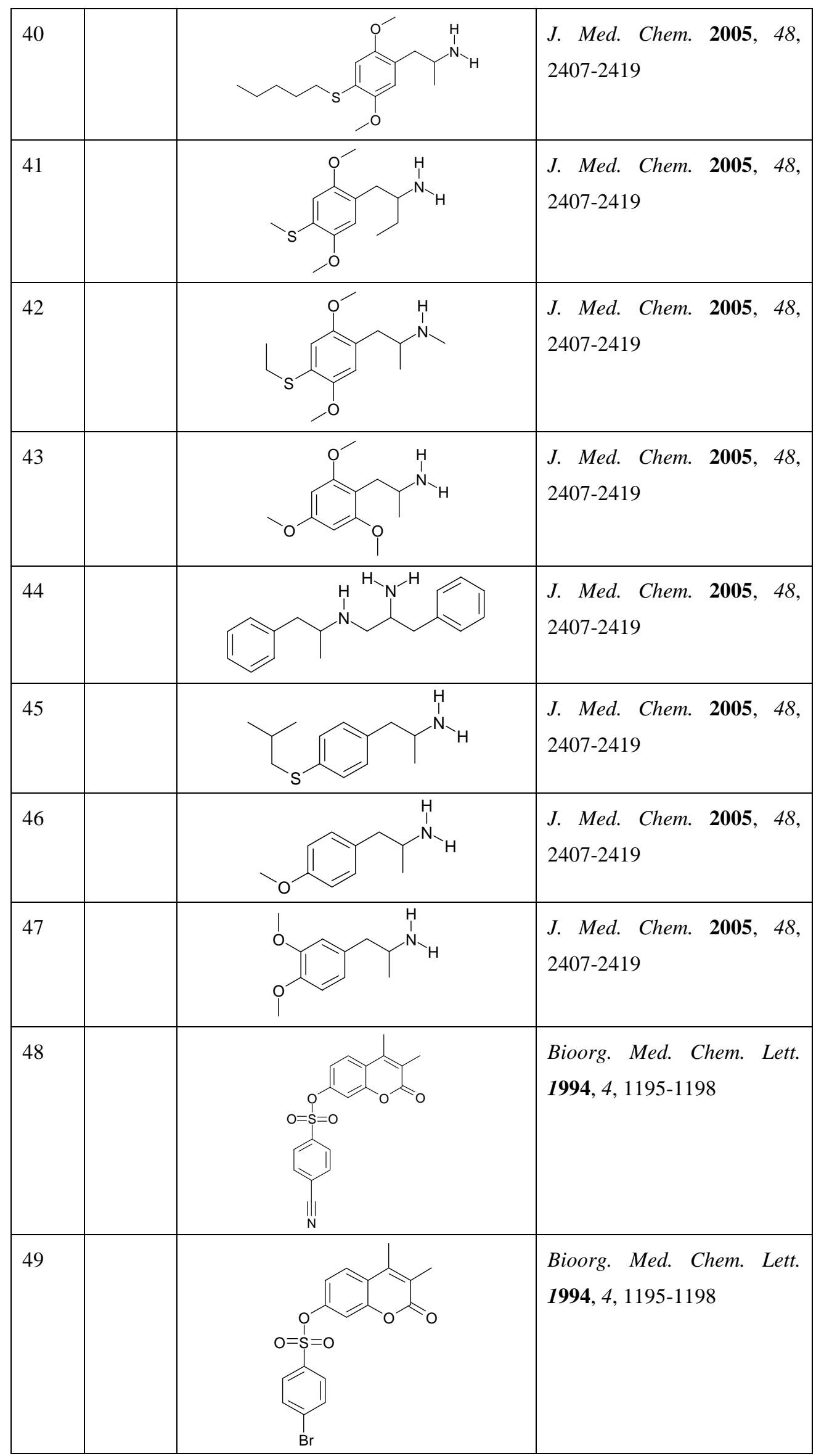




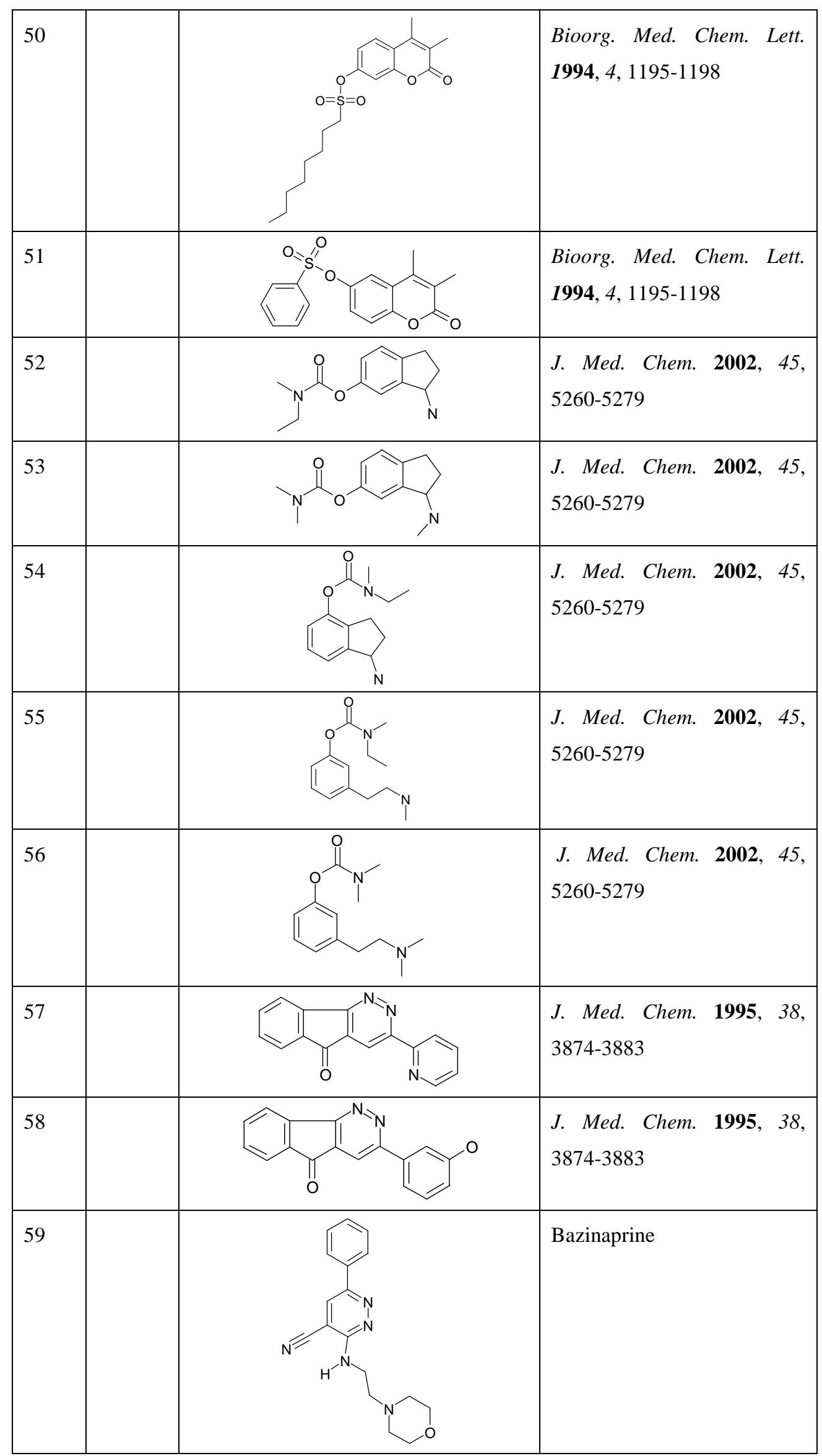




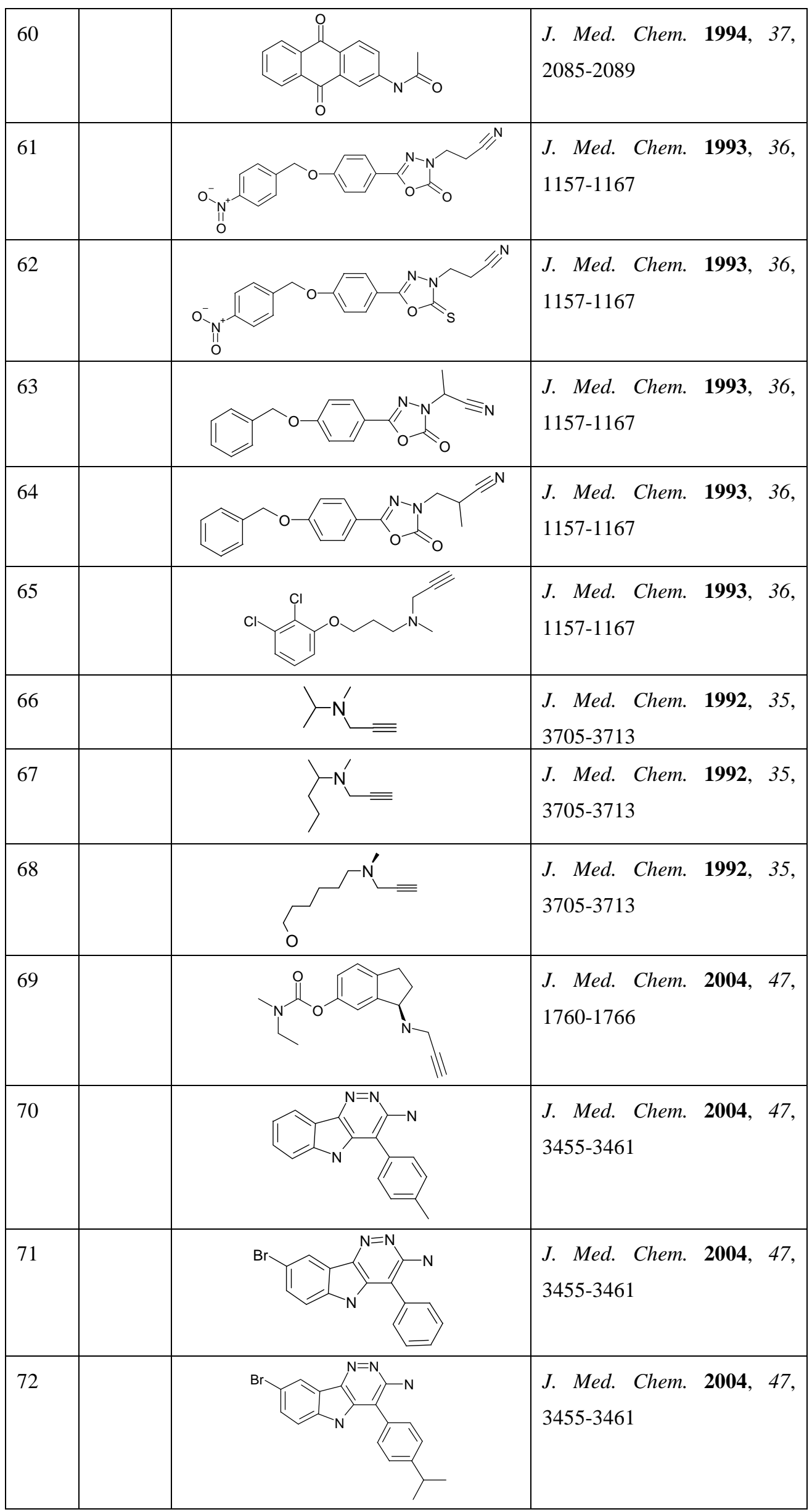




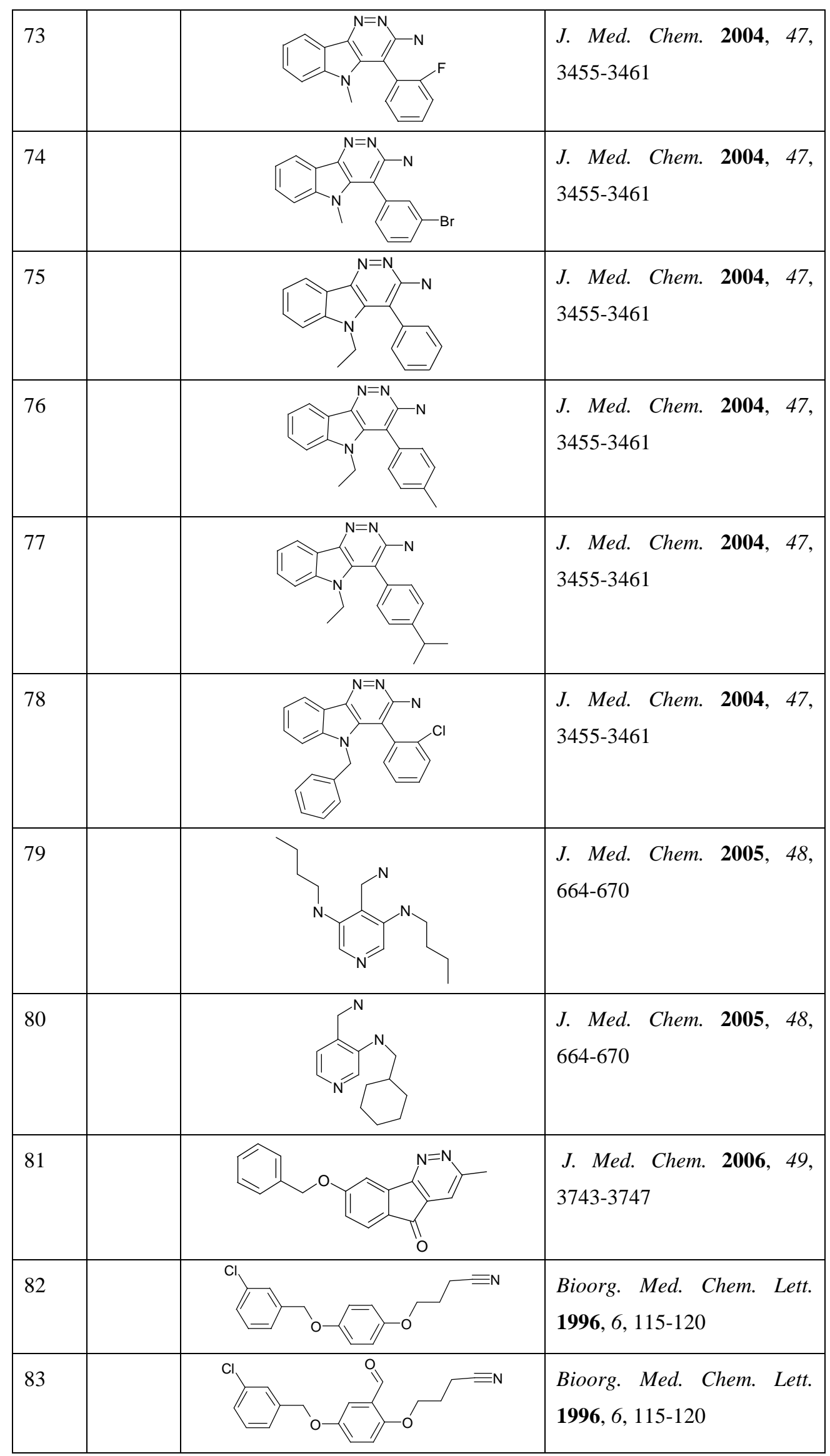




\begin{tabular}{|c|c|c|}
\hline 84 & o & $\begin{array}{l}\text { Bioorg. Med. Chem. Lett. } \\
\text { 1996, 6, 115-120 }\end{array}$ \\
\hline 85 & $F \mathcal{F}^{F}$ & $\begin{array}{l}\text { Bioorg. Med. Chem. Lett. } \\
\text { 1996, 6, 115-120 }\end{array}$ \\
\hline 86 & & $\begin{array}{l}\text { Bioorg. Med. Chem. Lett. } \\
\text { 1996, 6, 115-120 }\end{array}$ \\
\hline 87 & & $\begin{array}{l}\text { Bioorg. Med. Chem. Lett. } \\
\mathbf{1 9 9 6 , 6 , 1 1 5 - 1 2 0}\end{array}$ \\
\hline 88 & & $\begin{array}{l}\text { Bioorg. Med. Chem. Lett. } \\
\text { 1996, 6, 115-120 }\end{array}$ \\
\hline 89 & & $\begin{array}{l}\text { Bioorg. Med. Chem. Lett. } \\
\mathbf{1 9 9 6 , 6 , 1 1 5 - 1 2 0}\end{array}$ \\
\hline 90 & & $\begin{array}{l}\text { Bioorg. Med. Chem. Lett. } \\
\mathbf{1 9 9 6 , 6 , 1 1 5 - 1 2 0}\end{array}$ \\
\hline 91 & & $\begin{array}{l}\text { Bioorg. Med. Chem. Lett. } \\
\text { 1996, 6, 115-120 }\end{array}$ \\
\hline 92 & & $\begin{array}{l}\text { Bioorg. Med. Chem. Lett. } \\
\text { 1996, 6, 115-120 }\end{array}$ \\
\hline 93 & & $\begin{array}{l}\text { Bioorg. Med. Chem. Lett. } \\
\text { 1996, 6, 115-120 }\end{array}$ \\
\hline 94 & & $\begin{array}{l}\text { Bioorg. Med. Chem. Lett. } \\
\text { 1994, 4, 1195-1198 }\end{array}$ \\
\hline
\end{tabular}




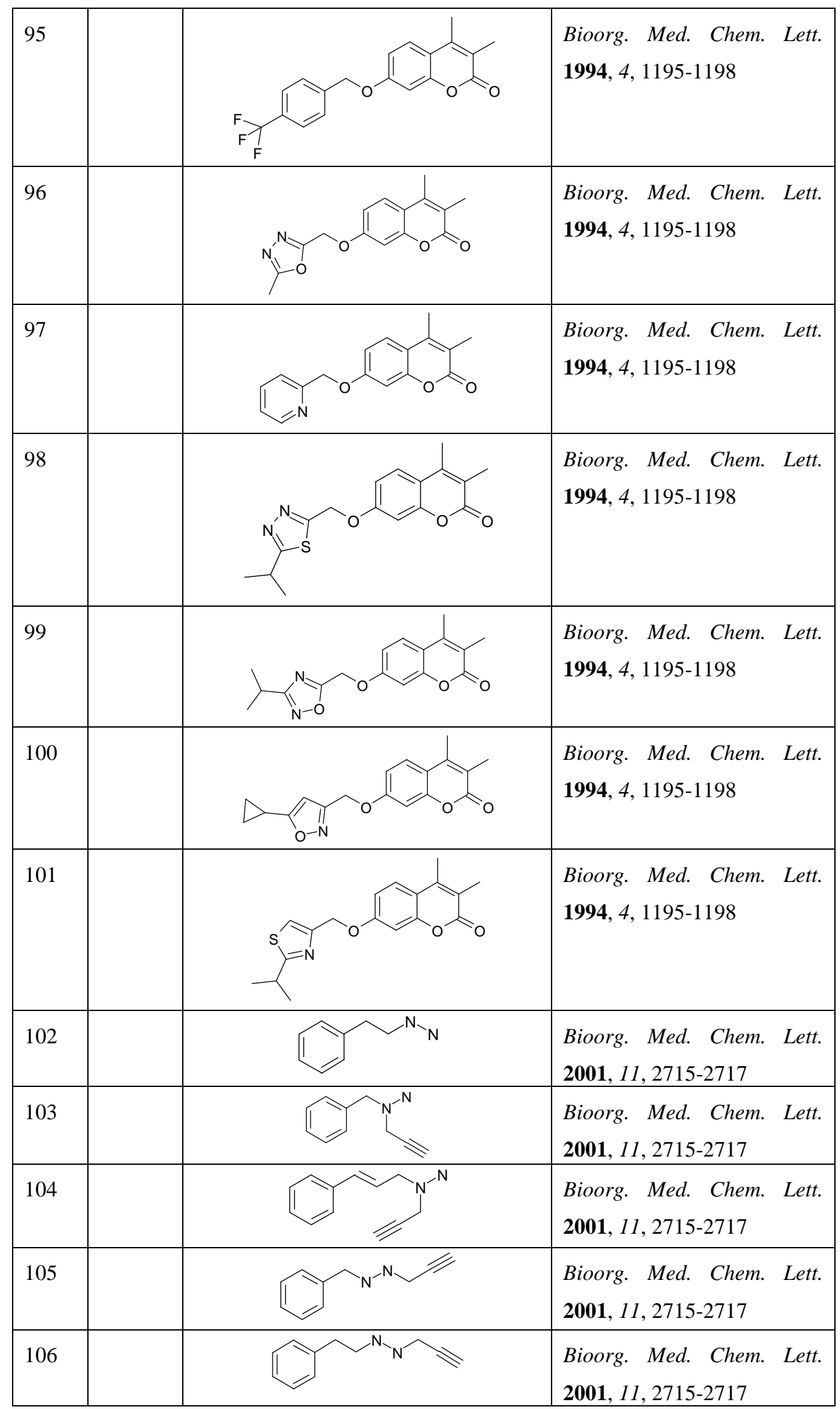




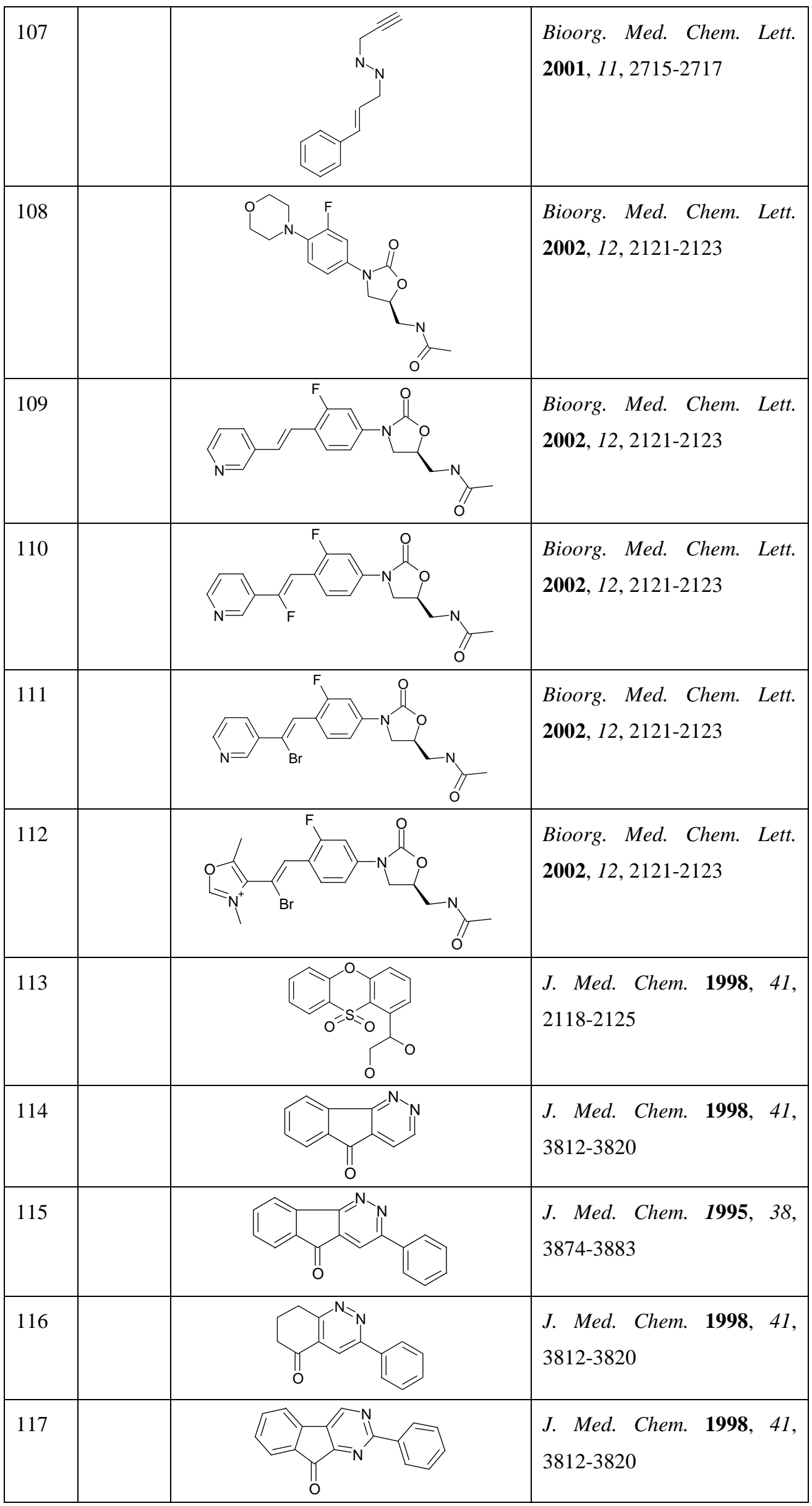


Int. J. Mol. Sci. 2007, 8

\begin{tabular}{|l|l|l|}
\hline 118 & $\begin{array}{l}\text { Bioorg. Med. Chem. 2004, } \\
\text { 12, 273-279 }\end{array}$ \\
\hline 119 & $\begin{array}{l}\text { Bioorg. Med. Chem. 2005, } \\
\text { 13, 773-783 }\end{array}$ \\
\hline 120 & \\
\hline 122 &
\end{tabular}

